\title{
Treatment of Allergic Diseases: Application to Clinical Practice of a New Concept of Mutual Substitutions of Antibody Molecules on the Surface of Mast Cells
}

Kimihiko Okazaki, $M D, P h D$

$T$ he concept of whether there are mutual substitutions of antibodies on cell surfaces has not received much attention to date, presumably because it does not look important. However, it could be far more relevant than first appearance because most, if not all, allergic diseases could be cured if nonpathogenic antibodies substituted pathogenic antibodies on the surface of mast cells.

In 1976, Turton reported that his hay fever had been cured after three time-repeated infections and exterminations of hookworm. ${ }^{1}$ In 1979, Kojima hypothesized in a domestic journal that antihookworm antibodies might have saturated all Fc receptors on Turton's mast cells. ${ }^{2}$

If there were only two antibody molecules on a cell, their mutual substitution could occur in a probability of $1 / 2$ because an equilibrium state exists between free and cellbound antibodies. If there were three antibody molecules, the probability would be $2 / 3$. It follows that when $\mathrm{N}$ antibody molecules are present, the probability of their mutual substitution is $(\mathrm{N}-1) / \mathrm{N}$. There are numerous antibody molecules bound to mast cells. Therefore, the probability, $(\mathrm{N}-1) / \mathrm{N}$, is virtually equal to 1 . A logical conclusion is that mutual substitutions of antibodies do occur on cell surfaces without fail.

Supporting the above theory are the results of the following case. In 1995, a 31-year-old woman with an egg allergy was treated with injections with a nonspecific antigen preparation, Asthremedin (Nippon Zohki Pharmaceutical Company, Osaka, Japan). Her blood samples were taken before and 4 months after the start of treatment. Ovalbumin and Asthremedin were separately labeled with fluorescence. Aliquots of her blood taken before the treatment were

Kimihiko Okazaki: Okazaki Medical Clinic, Kyoto, Japan.

Correspondence to: Dr. Kimihiko Okazaki, Okazaki Medical Clinic, 62 Azekatsucho, Nishikyogoku, Ukyoku, Kyoto, Japan 6150806; e-mail: ma13081x@ma1.seikyou.ne.jp.

DOI 10.2310/7480.2007.00019 separately incubated with labeled ovalbumin and labeled Asthremedin in an ice bath for 20 minutes. Ratios of leukocytes that became fluorescent during the incubation were analyzed using flow cytometry. Before treatment, the ratio of leukocytes that became fluorescent during the incubation with the labeled ovalbumin was $16.2 \%$ (mean of a duplicate test), whereas the ratio of leukocytes that became fluorescent during the incubation with labeled Asthremedin was $0.02 \%$ (mean of a duplicate test). After 4 months, the respective ratios were $9.6 \%$ and $17.4 \%$. These results demonstrate that there were substitutions of antibody molecules on the surface of leukocytes.

Repeated intradermal injections at 2- to 7-day intervals with a nonspecific antigen preparation, for example, killed microorganisms, may be a successful treatment of allergic disease. The appropriate initial dose would depend on the severity of the disease, that is, the more severe the disease, the less the initial dose. The least initial dose in my experience has been $1 \mathrm{ng}$ protein of killed Neisseria sicca et flava (Itoyu Pharmaceutical Company, Osaka, Japan). In most cases, 10 ng protein of killed Neisseria sicca et flava was used as the initial dose. The dose was raised by $50 \%$ after every 10 -time repetition. Mixing one-fifth of the volume with $1 \%$ lidocaine solution softened the pain accompanying the intradermal injection. The average necessary period of treatment has been one-thirteenth of the duration of the disease.

The total number of cases I have seen during the last 15 years is 721 , consisting of various allergies and atopic conditions. There has been no case of failure except for those who dropped out of the treatment. In addition, this treatment has caused no disagreeable side effects. The absence of failed cases implies that this treatment is reliable and deserves further study.

\section{References}

1. Turton JA. IgE, parasites, and allergy. Lancet 1976;2:686.

2. Kojima S. Parasite diseases, IgE and IgE antibodies. Rinsho-I 1979;5: 679. 